\title{
Nonholonomic Systems and Exponential Convergence: Some Analysis Tools
}

\author{
Robert T. M'Closkey* and Richard M. Murray ${ }^{\dagger}$ \\ Department of Mechanical Engineering \\ California Institute of Technology \\ Pasadena, CA 91125
}

\begin{abstract}
In this paper we make a contribution to the analysis of nonholonomic systems with exponential rates of convergence. A key idea is the use of control laws which render the closed loop system homogeneous with respect to a dilation. The analysis is applied to nonholonomic systems in power form and consists of two steps. The first step is a reduction to an invariant set and then the application of an averaging result. The averaging theorem is a stability result for $C^{0}$ homogeneous order zero rector fields.
\end{abstract}

\section{Introduction}

This paper develops some preliminary tools for analyzing and generating exponentially stabilizing control laws for systems of the form

$$
\dot{x}=\sum_{i=1}^{m} g_{i}(x) u_{i}(x, t) \quad x \in \mathbf{R}^{n},
$$

where each $g_{i}$ is a smooth vector field on $\mathbf{R}^{n}$ and the controls, $u_{i}(x, t)$ are continuous functions of $x$. Systems of this form arise in the study of mechanical systems with velocity constraints and have received renewed attention as an example of strongly nonlinear systems. See [12] for an introduction and more detailed motivation. For such systems, control methods based on linearization cannot be applied and nonlinear techniques must be utilized.

A control law $u=k(x, t)$ globally stabilizes a point $x_{0} \in \mathbf{R}^{n}$ if $x(t) \rightarrow x_{0}$ as $t \rightarrow \infty$ for all initial conditions of the system. For a nonholonomic control system, the dependence of a stabilizing control law on time is essential since the system (1) does not satisfy Brockett's necessary condition for smooth or even continuous stabilization [1] Hence there does not exist a smooth static state feedback law which stabilizes the system to a point. Recent work by Coron has shown that it is possible to stabilize a nonholonomic system using time-varying feedback $[2,3]$. Constructive approaches have been presented by Samson [17], Pomet [14], and Coron and Pomet [3].

All existing approaches based on Coron's results produce smooth asymptotic stabilizers which have very slow convergence rates (see [10] for an analysis). Stabilizing differentiable controls laws for driftless nonholonomic vector fields must necessarily decay at an algebraic rate (i.e. not exponential). This is easy to see because the linearized closed loop system is degenerate [13]. The algebraic rate of convergence generally decreases as the number of state variables increases over the number of control inputs. This is undesirable in practice since smal disturbances of the state can mean relatively long times for the state to return to some neighborhood of the equilibrium point. If decay rate of solutions to the equilibrium point is a measure of the system performance then this translates to poor performance. It can be shown that if exponential rates of convergence are desired, then the result contro law must be $C^{\alpha}$ with $0 \leq \alpha<1$. Coron has also proved the existence of continuous, time-varying controllers which are $C^{\infty}$ away from the origin and have exponential rates of convergence [3], but these controllers have not yet been exhibited on a sample system. In this paper we present a set of tools which are directed at generating continuous, time-periodic, controllers which result in exponential stability of nonholonomic systems. We apply these tools to some low-dimensional

\footnotetext{
'Supported in part by AFOSR F49620-92J-0293

'Supported in part by a grant from the Powell Foundation

0191-2216/93/\$3.00 @ 1993 IEEE
}

examples and demonstrate the existence of continuous exponential stabilizers for nonholonomic systems.

The techniques described in this paper are based on the study of of a special class of nonholonomic systems called chained systems, originally introduced in [12]. These systems highlight the geometric structure of the control system and have been very useful in the study of nonholonomic systems. We consider here a particular case and representation of a chained system which we call "power form" [18].

Systems in power form characterize the fundamental difficulties of nonholonomic systems in a very simple and useful way. By understanding the geometry of controllers applied to power form, we hope to understand the geometry of controllers applied to more general nonholonomic systems. This point of view has been used very successfully by Sussmann, who has shown how results applied to a "symbolic" representation of the control system can be used to understand systems with a compatible control Lie algebra [9].

Work of a similar spirit to ours is contained in Sørdalen's recent thesis [15] and a paper with Canudas de Wit [4]. They study exponential stabilization of car-trailer systems in the context of piecewise analytic feedbacks.

We study a specific class of continuous feedbacks, namely controls which render the closed loop vector field homogeneous with respect to a given dilation. The authors have derived such examples in modifying smooth stabilizing controls laws for nonholonomic systems in power form [10]. It is an open question as to what systems can be stabilized using homogeneous feedbacks. An undesirable feature of homogeneous vector fields is their coordinate dependent nature. This is somewhat perplexing since many important properties of control systems are coordinate independent (for example, nilpotentcy of the input vector fields). However, positive results for the low dimensional cases imply that homogeneous feedbacks warrant a closer look. Homogeneous vector fields have some nice properties,

1. they are "easier" to study,

2. a class of perturbations may be identified which does not affect the local asymptotic stability of the vector field.

The first statement deserves some clarification: the structure of homogeneous vector fields allows analysis in lower dimensions. This aspect and other properties are briefly reviewed in the next section. The second point has been demonstrated by Hermes for time-invariant vector fields [7]. It seems natural for this result to extend to time-varying homogeneous vector fields when the stability is uniform in $t$. This aspect is not pursued although we prove an averaging stability result for timeperiodic homogeneous vector fields. Before embarking on any general synthesis procedure for exponentially stabilizing controllers it is useful to have developed some analysis tools for the study of nonholonomic systems when the closed loop system is homogeneous. The three dimensional example examined in detail is used, along with a four and five dimensional example, to motivate the analysis tools presented in this paper.

The paper is organized as follows. Section 2 reviews dilations and some properties of homogeneous vector fields. Section 3 introduces the nonholonomic power form systems as well as the smooth stabilizing controllers from [18]. The approach which we take is to modify these smooth feedbacks and construct nondifferentiable control laws which render the system homogeneous relative to a certain dilation. Simulations of three, four and five dimensional power form systems with the modified control laws are presented and exhibit exponential con- 
vergence. A general method of analyzing the closed looped stability properties is proposed. The method consists of a reduction step and analysis of the corresponding lower dimensional system. The lower dimensional "reduced" vector field is still homogeneous and time periodic. Section 4 proves an averaging theorem which is applicable to the reduced vector fields obtained in the Section 3 . The reduction procedure is completely justified for the 3 dimensional case, however, these results are not included since the computations are long. The authors can provide details upon request.

\section{Dilations and Homogeneous Norms}

Let $\mathcal{V}$ be a real finite dimensional vector space of dimension $n$. Suppose a basis has been chosen for $\mathcal{V}$ such that its elements are represented by the following $n$-tuple:

$$
x=\left(x_{1}, x_{2}, \ldots, x_{n}\right) .
$$

A dilation is defined by assigning $n$ positive rationals $r=\left(1 \leq r_{1} \leq\right.$ $r_{2} \leq \cdots \leq r_{n}$ ) and the following map $\delta_{\lambda}{ }^{r}: \mathcal{V} \rightarrow \mathcal{V}$,

$$
\delta_{\lambda}{ }^{r} x=\sum_{i=1}^{n} \lambda^{r_{i}} x_{i}, \quad \lambda>0
$$

In most applications the $r_{i}$ 's are integers and the $r$ is dropped from from the notation.

Definition 1 A continuous function $f: \mathbf{R}^{n} \rightarrow \mathbf{R}$ is homogeneous of degree $l \geq 0$ with respect to $\delta_{\lambda}{ }^{\top}$, denoted $f \in H_{l}$ if $f\left(\delta_{\lambda}{ }^{\top} x\right)=\lambda^{l} f(x)$. A continuous vector field $X$ on $R^{n}$ is homogeneous of degree $m \leq 1$ with respect to $\delta_{\lambda}$ if $X f \in B_{j-m}$ whenever $f$ is smooth and $f \in H_{j}$.

We will take $\mathcal{V}=\mathbf{R}^{n}$ with the usual vector space structure for the problems studied here. The standard dilation $\left(r_{i}=1, i=1, \ldots, n\right)$ on $R^{n}$ warps the space isotropically, that is it stretches each coordinate direction the same amount.

Definition 2 A continuous map from $\mathbf{R}^{n}$ to $R, x \rightarrow \rho(x)$, is called a homogeneous norm with respect to the dilation $\delta_{\lambda}$ when

1. $\rho(x) \geq 0, \quad \rho(x)=0 \Longleftrightarrow x=0$,

2. $\rho\left(\delta_{\lambda} x\right)=\lambda \rho(x) \quad \forall \lambda>0$.

Calling this function a "norm" is a misnomer since it need not satisfy the triangle inequality. However, the triangle inequality is not required to define convergence of a sequence. The important property of $\rho$ is its positive definiteness. The topology on $R^{n}$ induced by $\rho(\cdot)$ is equivalent to the topology induced by any of the usual p-norms. We are primarily interested in the convergence of time dependent functions using the homogeneous norm as a measure of their size. When a vector field is homogeneous it is most natural to use a corresponding homogeneous norm as the metric.

The concept of exponential stability of a vector field is now introduced in the context of a homogeneous norm. This definition was introduced by Kawski [8]. Let $f(t, x)$ be a continuous vector function of its arguments,

$$
f(t, x): \mathbf{R} \times \mathbf{R}^{n} \rightarrow \mathbf{R}^{n} .
$$

Without loss of generality we assume that $x=0$ is an isolated equilibrium point of the system, $f(t, 0)=0, \forall t$. A solution of the equations passing through $x_{0}$ at time $t_{0}$ is represented by $x\left(t, x_{0}, t_{0}\right)$.

Definition 3 The equilibrium point $x=0$ is locally exponentially stable with respect to the homogeneous norm $\rho(\cdot)$ if there exist two strictly positive numbers $\alpha$ and $\beta$ such that

$$
\rho\left(x\left(t, x_{0}, t_{0}\right)\right) \leq \alpha \rho\left(x_{0}\right) e^{-\beta\left(t-t_{0}\right)} \quad \forall t \geq t_{0},
$$

provided $\rho\left(x_{0}\right)$ is sufficiently small.

We will see that this notion of stability is important when considering vector fields which are homogeneous with respect to a dilation. The convergence of trajectories is naturally studied using the corresponding homogeneous norm. The standard results on local exponential stability may be interpreted in this framework: when the linearization is defined, the "correct" dilation to employ is the standard dilation and the homogeneous norm reduces to any $L_{p}$ norm on $\mathbf{R}^{n}$.

Furthermore, the following are equivalent for homogeneous vector fields of order zero:

$$
\begin{aligned}
& \text { 1. } x=0 \text { is locally uniformly asymptotically stable with respect } \\
& \text { to } \rho(\cdot) \text {, }
\end{aligned}
$$

2. $x=0$ is globally exponentially stable with respect to $\rho$.

This fact is stated in [8] for the time-invariant case and also holds for time-varying vector fields.

Lastly, some important aspects of homogeneous order zero vector fields are now presented. The action of the dilation on a solution of (2) satisfies

$$
x\left(t, t_{0}, \delta_{\lambda}\left(x_{0}\right)\right)=\delta_{\lambda}\left(x\left(t, t_{0}, x_{0}\right)\right) .
$$

Furthermore, the dilation $\delta_{\lambda}$ specifies a transformation group on the phase space which facilitates the study of equation (2) by considering a vector field on the quotient manifold specified by the group. The quotient manifold is given by $\rho(x)=1$ which is just a warped sphere, $S^{n-1}$, naturally embedded in $R^{n}$. The projection map $\pi: R^{n} \backslash\{0\} \rightarrow$ $S^{n-1}$ onto the sphere is

$$
\pi(x)=\left(\frac{x_{1}}{\rho^{r_{1}}(x)}, \frac{x_{2}}{\rho^{r_{2}}(x)}, \ldots, \frac{x_{n}}{\rho^{r_{n}}(x)}\right) .
$$

Pushing forward the vector field with this map defines a unique vector field on the sphere by virtue of the transformation group invariance. Once the flow on the sphere is known the flow of the original vector field is determined by lifting the sphere flow with an additional scalar equation. The scalar equation is merely the differential equation for $\rho$ written in the sphere coordinates. Determining the asymptotic behavior of the full set of equations amounts to computing the nonwandering set, $\Omega$, on the sphere and observing the behavior of $\rho$ on $\Omega$. Full details of the time invariant case are found in [8]. Related work for the time-varying case is in [10].

\section{Examples and Analysis}

We will study a three dimensional example in detail and briefly present some higher dimensional cases. The nonholonomic systems studied are those in power form,

$$
\begin{aligned}
\dot{x}_{1} & =u_{1} \\
\dot{x}_{2} & =u_{2} \\
\dot{x}_{3} & =x_{2} u_{1} \\
\dot{x}_{4} & =\frac{1}{2} x_{2}^{2} u_{1} \\
& \vdots \\
\dot{x}_{n} & =\frac{1}{(n-2) !} x_{2}^{n-2} u_{1} .
\end{aligned}
$$

The control Lie algebra for this system is spanned by the input vector fields and Lie products of the form $\mathrm{ad}_{g_{1}}^{k} g_{2}$. Necessary and sufficient conditions for converting a system into power form are given in [11] All two-input nonholonomic systems in $R^{3}$ and $R^{4}$ are locally feedback transformable to power form. In the sequel, the origin is always taken to be the point that is stabilized.

The starting point for our analysis is modification of the smooth stabilizing control laws derived by Teel et al. [18],

$$
\begin{aligned}
& u_{1}=-x_{1}-\sum_{j=1}^{n-2} c_{j} x_{j+2} \cos j t, \\
& u_{2}=-x_{2}-\sum_{j=1}^{n-2} x_{j+2}^{2}(\sin t-\cos t),
\end{aligned}
$$

with $c_{j}>0$ (actually, we have switched the roles of $x_{1}$ and $x_{2}$ in that reference). In [10] we used the following general form for the 3-dimensional system control law,

$$
\begin{aligned}
& u_{1}=-x_{1}+F_{1}\left(x_{3}\right) \cos t \\
& u_{2}=-x_{2}+F_{2}\left(x_{3}\right) \sin t .
\end{aligned}
$$

This control is essentially the same as the feedback used by Teel et al. the only difference being the phases of the time periodic terms (the relative phases amongst the terms remains the same). We showed in [10] that, based on a simple multiscale perturbation argument, the convergence of $x_{3}$ is controlled by the product of $F_{1}$ and $F_{2}$. The reasoning behind this heuristic analysis is as follows: if $x_{3}$ evolves on a much slower time scale than $x_{1}$ or $x_{2}$ then we may take $x_{3}$ equal to a constant in the $\dot{x}_{1}$ and $\dot{x}_{2}$ equations. We then solve for $x_{1}$ and $x_{2}$ as 
a function of $x_{3}$ and substitute these expressions into the $\dot{x}_{3}$ equation to determine its stability. Of course this analysis is nothing more than center manifold theory when the $F_{i}$ 's are $C^{2}$. The perturbation analysis reveals the $\dot{x}_{3}$ equation to have the form,

$$
\dot{x}_{3}=-\frac{\epsilon}{4} F_{1}\left(x_{3}\right) F_{2}\left(x_{3}\right) \text {. }
$$

We choose $F_{1}=\operatorname{sgn}\left(x_{3}\right) \sqrt{\left|x_{3}\right|}$ and $F_{2}=\sqrt{\left|x_{3}\right|}$ so that their product is $x_{3}$. An important fact to note is that the 3-D closed loop vector field is invariant under the group action,

$$
\left(x_{1}, x_{2}, x_{3}\right) \stackrel{\delta_{\lambda}}{\rightarrow}\left(\lambda x_{1}, \lambda x_{2}, \lambda^{2} x_{3}\right) \quad \lambda>0 .
$$

Choosing a homogeneous norm,

$$
\rho(x)=\left(x_{1}^{4}+x_{2}^{4}+x_{3}^{2}\right)^{\frac{1}{4}},
$$

we were able to derive the equations on the sphere $\rho(x)=1$. We numerically proved for the attractive sets on the sphere that all solutions back in the full phase space, corresponding to these sets, were exponentially stable with respect to the homogeneous norm. However, it was noted that identifying the entire $\Omega$-limit set could be difficult. For instance, an exhaustive numerical study would be required to rigorously identify $\Omega$. This may be satisfactory for specific problems but if one is interested in proving general results the numerical approach cannot be used.

Another interesting fact which is currently being explored is how to choose the "right" dilation. In the 3-D example we chose the form of the feedback based on perturbation analysis. Results by Gurvitz and $\mathrm{Li}$ seem to indicate that the inverse of the degree of nonholonomy (the smallest order of brackets required for controllability) is an upper bound for the Hölder continuity of the control law [5]. Our examples discussed below achieve this bound. The powers of the dilation we choose for these examples are intimately connected with the controllability growth vector of each system. The intuition behind this last statement is as follows: suppose it takes a certain number of Lie brackets before a given direction in the control Lie algebra is spanned, then it seems reasonable to give the states spanning that new direction a weighted value corresponding to the bracket number since moving in that direction is "harder" than moving in a direction spanned by fewer brackets; if exponential stability is desired, then the weight forces a certain Hölder continuity to keep the vector field homogeneous of order zero.

The growth vector for the 3-D power system is $(2,1)$. Thus, the first two states can be directly controlled through the inputs and the last state direction is spanned after one level of brackets so the vector of powers in the dilation is taken to be $\left(r_{1}, r_{2}, r_{3}\right)=(1,1,2)$. Similarly, vectors for the 4 - and 5 -D power systems are $(1,1,2,3)$ and $(1,1,2,3,4)$, respectively. Hence the 4-D dilation to is,

$$
\delta_{\lambda}\left(x_{1}, x_{2}, x_{3}, x_{4}\right)=\left(\lambda x_{1}, \lambda x_{2}, \lambda^{2} x_{3}, \lambda^{3} x_{4}\right),
$$

and the 5-D dilation

$$
\delta_{\lambda}\left(x_{1}, x_{2}, x_{3}, x_{4}, x_{5}\right)=\left(\lambda x_{1}, \lambda x_{2}, \lambda^{2} x_{3}, \lambda^{3} x_{4}, \lambda^{4} x_{5}\right) .
$$

Now in order to modify the smooth controls (5) intelligently we will use the previous dilations to determine the Hölder exponent of the individual terms in the control law which makes the closed loop vector field homogeneous of order zero. In addition, we will respect the algebraic sign of each term. We also take $c_{i}=\epsilon$ for all $i$. When $\epsilon=0, x_{1} \rightarrow 0$ and the $x_{i}$ 's, for $i=3,4 \ldots$, limit to constant values. This set is an attractive invariant set for the closed loop system. Near this set $x_{i}$, $i=1,3,4 \ldots$, vary slowly. When $0<\epsilon<1$, it is quite possible that a perturbed form of the set persists. This fact is proven by the anthors in an extended version of this paper for the 3-D power form system with the feedback described below. The modified feedbacks are, for the 3-D system,

$$
\begin{aligned}
& u_{1}=-x_{1}-\operatorname{\epsilon ggn}\left(x_{3}\right) \sqrt{\left|x_{3}\right|} \cos t, \\
& u_{2}=-x_{2}-\sqrt{\left|x_{3}\right|}(\sin t-\cos t),
\end{aligned}
$$

the 4-D system,

$$
\begin{aligned}
& u_{1}=-x_{1}-\epsilon\left(\operatorname{sgn}\left(x_{3}\right) \sqrt{\left|x_{3}\right|} \cos t+\operatorname{sgn}\left(x_{4}\right)\left|x_{4}\right|^{\frac{1}{3}}\right) \\
& u_{3}=-x_{2}-\left(\sqrt{\left|x_{3}\right|}+\left|x_{4}\right|^{\frac{1}{3}}\right)(\sin t-\cos t),
\end{aligned}
$$

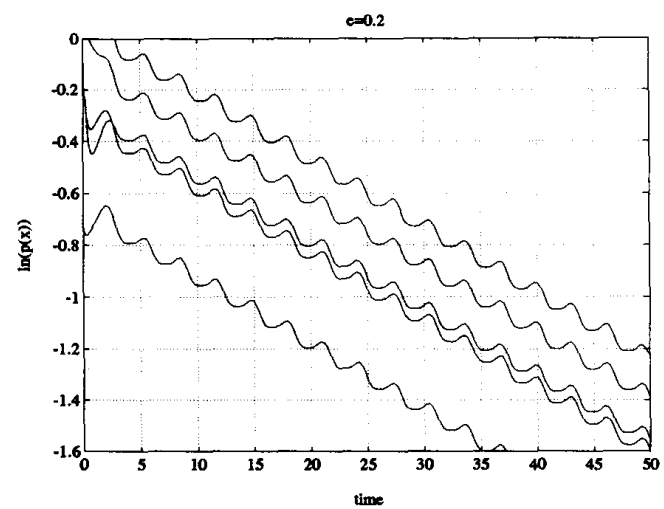

Figure 1: Exponential Rates of Convergence of 3-D System

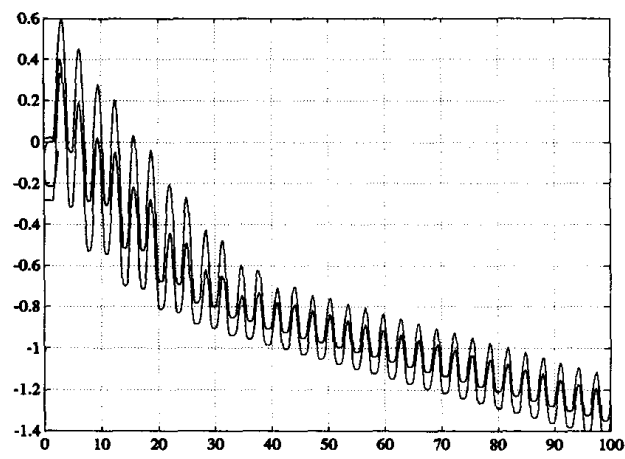

Figure 2: Exponential Rates of Convergence of 4-D System and the 5-D system,

$$
\begin{aligned}
& u_{1}=-x_{1}-\epsilon\left(\operatorname{sgn}\left(x_{3}\right) \sqrt{\left|x_{3}\right|} \cos t+\operatorname{sgn}\left(x_{4}\right)\left|x_{4}\right|^{\frac{1}{3}}+\operatorname{sgn}\left(x_{5}\right)\left|x_{5}\right|^{\frac{1}{4}}\right) \\
& u_{2}=-x_{2}-\left(\sqrt{\left|x_{3}\right|}+\left|x_{4}\right|^{\frac{1}{2}}+\left|x_{5}\right|^{\frac{1}{t}}\right)(\sin t-\cos t) .
\end{aligned}
$$

Simulations with random initial conditions in a ball are presented in Figures 1 to 3 . The $\log$ of the homogeneous norm of the states versus time is plotted. The fact the the plots are bounded above by a straight line implies exponential stability. For the 5-D case this is difficult to see because there are multiple rates of decay, some of them very slow. However, the solutions are certainly asymptotically stable (this fact will be transparent once the proof in Section 4 is understood) and since the vector field is periodic in $t$, the stability is uniform. Hence, the states are converging exponentially to the origin (see the review of homogeneous systems). The arguments presented above have some merit and we endeavor to show why they are true.

In terms of an analysis procedure, what can be done to rigorously determine the stability of the closed loop system and, if possible, obtain estimates on the exponential rates of convergence? We are proposing these systems may be studied using a two-step procedure:

1. first a "reduction" where $x_{1}$ and $x_{2}$ are approximately represented in terms of the remaining states,

2. then a stability analysis of the reduced $n-2$ dimensional system.

The most desirable result would be stability of the reduced system implying similar behavior of the original system. When the vector field is at least $C^{2}$ this two-step analysis is nothing more than singular perturbation theory (when the equilibrium point is hyperbolic) or center manifold theory (when the equilibrium point posses a center eigenspace). For these cases, if the invariant manifold is stable, stability of the original system is determined by the stability of its reduced 


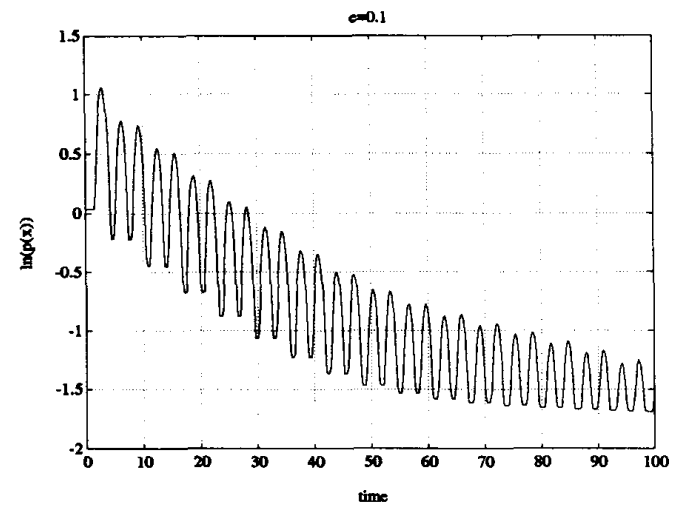

Figure 3: Exponential Rates of Convergence of 5-D System

part. However, we can never hope to have a differentiable vector field, so applying these well known results is not possible without further analysis.

Suppose we proceed with the heuristic analysis mentioned described above and consider $x_{i}, i=3,4, \ldots$, fixed in the $x_{1}$ and $x_{2}$ equations. The following general form of $x_{1}$ and $x_{2}$ is arrived at,

$$
\begin{aligned}
& x_{1}=\sum_{j=1, \ldots, n-2} \frac{\epsilon}{1+j^{2}} \operatorname{sgn}\left(x_{j+2}\right)\left|x_{j+2}\right|^{j+1}(-j \sin j t-\cos j t) \\
& x_{2}=\sum_{j=1, \ldots, n-2}\left|x_{j+2}\right|^{\frac{1+1}{1+1}}(\cos t) .
\end{aligned}
$$

Substituting (12) into the higher order equations for $x_{i}, i \geq 3$,

$$
\begin{aligned}
\dot{x}_{i}= & \frac{1}{(i-2) !} x_{2}^{i-2} u_{1} \\
= & \frac{1}{(i-2) !}\left(\sum_{j=1, \ldots, n-2}\left|x_{j+2}\right|^{\frac{1}{j+1}} \cos t\right)^{i-2} . \\
& \sum_{j=1}^{n-2} \frac{j \epsilon}{1+j^{2}} \operatorname{sgn}\left(x_{j+2}\right)\left|x_{j+2}\right|^{j+1}(\sin j t-j \cos j t)
\end{aligned}
$$

The systems of equations formed by (13) are homogeneous with respect to the same dilation with $x_{1}$ and $x_{2}$ eliminated. Furthermore, the reduced equations are periodic with period $2 \pi$. If we average the reduced vector field over one period the reader can check the following result,

$$
\dot{\bar{x}}_{i}=-\epsilon \frac{(i-2)^{2}}{2(i-2) !\left(1+(i-2)^{2}\right)}\left(\sum_{j=1, \ldots, n-2}\left|\bar{x}_{j+2}\right|^{\frac{3+1}{3}}\right)^{i-2} \operatorname{sgn}\left(\bar{x}_{i}\right)\left|\bar{x}_{i}\right|^{\frac{1}{i-1}}
$$

for $i \geq 0$. The overline denotes the averaged variable. The averaged set of reduced equations (14) is asymptotically stable by inspection since the "coefficient" in front of the $\operatorname{sgn}\left(\bar{x}_{i}\right)\left|\bar{x}_{i}\right|^{\frac{1}{i-1}}$ term is positive definite on the reduced phase space. Now we may apply the "averaging theorem" proven in Section 4 to conclude exponential stability of the original set of reduced equations (13) for $\epsilon$ sufficiently small. At this point, we would like to conclude exponential stability of the full set of equations (4) with the feedbacks (9) to (11) and their higher dimensional extensions. The initial reduction procedure has not been rigorously justified. However, the simulations and averaging analysis strongly suggest that the reduction results are correct, at least for determining the stability of the system. A proof for the 3-D power form system with the feedback given by (9) is available from the first author.

\section{Averaging Results for Homogeneous Sys- tems}

In this section we present an averaging result which will be useful for analyzing the closed loop equations. First we introduce the class of systems of interest. Consider the differential equation

$$
\dot{x}=\epsilon f(t, x, \epsilon),
$$

where $f \in C\left(\mathbf{R} \times \mathbf{R}^{n} \times I, \mathbf{R}^{n}\right), C\left(\mathbf{R} \times \mathbf{R}^{n} \times I, \mathbf{R}^{n}\right)$ being the set of continuous map from $\mathbf{R}^{n} \times \mathbf{R}^{n} \times I$ into $\mathbf{R}^{n} . f$ is periodic of period $T$ with respect to $t$ and $f(t, 0, \epsilon)=0$ for all $t$ in $(-\infty, \infty)$. We will rescale time so that the period is always $2 \pi$. We further restrict our attention to a class of homogeneous vector fields. The vector field in (15) is homogeneous of order zero with respect to the dilation $\delta_{\lambda}=$ $\left(\lambda^{r_{1}} x_{1}, \lambda^{r_{2}} x_{2}, \ldots, \lambda^{r_{n}} x_{n}\right)$. A solution of (15) through the point $x_{0}$ at time $t_{0}$ is denoted $x\left(t, t_{0}, x_{0}\right)$.

Before moving to the averaging result we give an equivalent definition of asymptotic stability of a fixed point of a general differential equation with continuous vector field,

$$
\dot{x}=g(t, x),
$$

where $g(t, 0)=0$ for all $t$. The solution $x=0$ of $(16)$ is asymptotically stable if it is stable and if there exists a $\delta=\delta\left(t_{0}\right)$, and for all $\epsilon>0$ there exists a $\tilde{t}\left(\epsilon, t_{0}\right)$, such that $\left|x_{0}\right|<\delta$ implies $\left|x\left(t, t_{0}, x_{0}\right)\right|<\epsilon$ for all $t>\tilde{t}$ This is easily shown from the usual definition of asymptotic stability.

In the averaging theorem we will infer stability (instability) of the zero solution of equation (15) from stability (instability) of the zero solution of the averaged system,

$$
\dot{x}=\epsilon f_{0}(x),
$$

where

$$
f_{0}(x)=\lim _{T \rightarrow \infty} \frac{1}{T} \int_{0}^{T} f(t, x, \epsilon) d t .
$$

The vector field in (15) is $2 \pi$-periodic in $t$ so the average in (18) is equivalent to

$$
f_{0}(x)=\frac{1}{2 \pi} \int_{0}^{2 \pi} f(t, x, 0) d t .
$$

Note that $f_{0}$ is homogeneous of order zero with respect to the dilation $\delta_{\lambda}$. Before the averaging result is stated we prove another lemma.

Define the one-parameter family of diffeomorphisms on the extended phase space of equation (15) which leave it invariant,

$$
\begin{aligned}
\Psi_{\lambda}: S^{1} \times \mathbf{R}^{n} & \rightarrow S^{1} \times \mathbf{R}^{n} \\
(t, x) & \mapsto\left(t, \delta_{\lambda}(x)\right) \quad \lambda>0 .
\end{aligned}
$$

We also define three nested homogeneous balls in the extend phase space

$$
B_{i}=\left\{(t, x) \in S^{1} \times R^{n} \mid \rho(x) \leq c_{i}\right\} \quad i=\{1,2,3\},
$$

where $c_{1}>c_{2}>c_{3}>0$.

Lemma 4.1 (Scaling Lemma). For time periodic homogeneous order zero vector fields (15) and given the $B_{i}$ 's defined above, stppose we know the following facts,

1. $\left(t_{0}, x_{0}\right) \in B_{2}$ implies $\left(t, x\left(t, t_{0}, x_{0}\right)\right) \in B_{1}$ for all $t>t_{0}$,

2. there exists a $\bar{T}>0$ such that $\left(t_{0}, x_{0}\right) \in B_{2}$ implies $\left(t, x\left(t, t_{0}, x_{0}\right)\right) \in B_{3}$ for all $t>\bar{T}$.

Then the zero solution of (15) is asymptotically stable.

Proof. We first prove stability. Start (15) with initial conditions in $\boldsymbol{B}_{2}$. Then $\left(t, x\left(t, t_{0}, x_{0}\right)\right) \in B_{1}$ for all $t>t_{0}$. In other words, $\rho\left(x_{0}\right)<c_{2}$ implies $\rho\left(x\left(t, t_{0}, x_{0}\right)\right)<c_{1}$ for all $t>t_{0}$. This condition may be extend to any neighborhood using the mapping $\delta_{\lambda}$. Suppose the bound $\rho\left(x\left(t, t_{0}, x_{0}\right)\right)<e_{1}$ for all $t>t_{0}$ is desired, then restrict $\rho\left(x_{0}\right)<e_{1} \frac{c_{2}}{c_{1}}$. Specifically,

$$
\begin{aligned}
x\left(t, t_{0}, x_{0}\right) & =x\left(t, t_{0}, \delta_{e_{1} / c_{1}}\left(\delta_{c_{1} / e_{1}}\left(x_{0}\right)\right)\right) \\
& =\delta_{e_{1} / c_{1}}\left(x\left(t, t_{0},\left(\delta_{c_{1} / e_{1}}\left(x_{0}\right)\right)\right)\right)
\end{aligned}
$$

but,

$$
\rho\left(\delta_{c_{1} / e_{1}}\left(x_{0}\right)\right)=\frac{c_{1}}{e_{1}} \rho\left(x_{0}\right)<\frac{c_{1}}{e_{1}} \frac{c_{2}}{c_{1}} e_{1}=c_{2} .
$$

Hence,

$$
\begin{aligned}
\rho\left(x\left(t, t_{0}, x_{0}\right)\right) & =\rho\left(\delta_{e_{1} / c_{1}}\left(x\left(t, t_{0}, \delta_{c_{1} / e_{1}}\left(x_{0}\right)\right)\right)\right. \\
& =\frac{e_{1}}{c_{1}} \rho\left(x\left(t, t_{0}, \delta_{\frac{e_{1}}{e_{1}}}\left(x_{0}\right)\right)\right) \\
& =e_{1} .
\end{aligned}
$$

This is stability of the zero solution. See Figure 4 for a picture. 


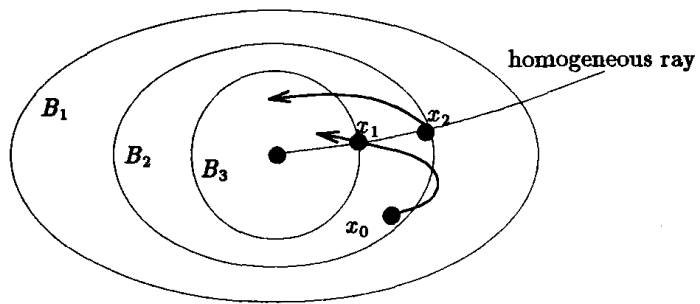

Figure 4: Homogeneous balls used in proof: use the dilation to map $x_{2}$ to $x_{1}$ thus extending the trajectory starting at $x_{0}$.

To demonstrate asymptotic stability we proceed in a similar manner. Define the annulus,

$$
A_{2}=B_{2} \backslash B_{3} .
$$

We know that solutions with initial conditions in $A_{2}$ enter $B_{3}$ in finite time $\bar{T}$ and remain there. Since the differential equation is invariant under $\Psi$ then we may map the annulus $A_{2}$ to another annulus that sits inside $A_{2}$ and shares a common boundary. In this way solutions starting in $A_{2}$ are extended into the new annulus (because of the invariance) and can only remain in the new annulus for a finite time. Define the sequence of nested annuli.

$$
A_{i}=\Psi_{c_{3} / c_{z}}\left(A_{i-1}\right), \quad i=3,4, \ldots
$$

Note that the outer boundary of $A_{i}$, denoted $\partial^{\circ} A_{i}$, is the inner boundary of $A_{i-1}$, denoted $\partial^{i} A_{i-1}$, because,

$$
\begin{aligned}
\partial^{\circ} A_{i} & =\Psi_{c_{3} / c_{2}}\left(\partial^{0} A_{i-1}\right) \\
& =\Psi_{\left(c_{3} / c_{3}\right)^{i-2}}\left(\partial^{0} A_{2}\right) \\
& =\Psi_{\left(c_{3} / c_{2}\right)^{i-*}}\left(\Psi_{c_{3} / c_{2}}\left(\partial^{o} A_{2}\right)\right) \\
& =\Psi_{\left(c_{3} / c_{2}\right)^{i-3}}\left(\partial^{i} A_{2}\right) \\
& =\partial^{i} A_{i-1} .
\end{aligned}
$$

The properties of solutions with initial conditions in $A_{2}$ are shared by the other annuli. Hence, an initial condition in $A_{i}$ must enter $A_{i+1}$ in time $\bar{T}$. Extending this to the larger annulus defined by

$$
\Sigma_{N}=\bigcup_{i=2, \ldots, N} A_{i}, \quad N>2
$$

implies that solutions with initial conditions here will enter the set $A_{N+1}$ in a time no less that $N \bar{T}$ and can never reenter $\Sigma_{N}$. Thus we pick $\delta=c_{2}$ and for $\epsilon>0$ choose $\bar{t}=m \bar{T}$ where $m$ satisfies $\left(\frac{c_{2}}{c_{2}}\right)^{m}<\epsilon$. This is equivalent to asymptotic stability.

Remark This lemma actually demonstrates exponential stability of the zero solution becasse the time taken to leave any given annulus is independent of the "size" of the annulus (this is a result of the vector field having degree zero with respect to the dilation $\delta_{\lambda}$ ). At time $t>m \bar{T}$ any solution may be bounded by a homogeneous ball with size proportional to $\left(\frac{c_{2}}{c_{2}}\right)^{m}$. Hence, this bound plus stability of the solutions may be recast as an exponential stability result with respect to the homogeneous norm $\rho$.

Theorem 4.1 Consider the equations (15). Suppose $x=0$ is an asymptotically stable fixed point of the averaged system $\dot{y}=\epsilon f_{0}(y)$ Then for $\epsilon>0$ sufficiently small, the solution $x=0$ is exponentially stable for the full equations (15).

This result is already well known for $C^{1}$ vector fields where $x=0$ is a hyperbolic fixed point. Proving the theorem when the vector field is differentiable is straight forward since the standard averaging change of coordinates places the vector field into a form where the time-varying part is bounded with an arbitrarily small Lipschitz constant (by making $\epsilon$ sufficiently small). Hence, if $x=0$ is a hyperbolic fixed point of $f_{0}$ then the stability of the full system is determined by the stability of $f_{0}$ for $\epsilon$ sufficiently small. Unfortunately this proof does not extend to our case since the averaging change of coordinates tends to "mix" the new coordinates so that the transformed vector field is no longer homogeneous. However we may get a total stability result in the new coordinates which will imply certain strong behavior of the solutions of the original homogeneous system. The idea of the proof uses the fact that in the new coordinates we may choose $\epsilon$ small enough so that we may make a ball about the origin attractive and invariant. Mapping this ball back to the original coordinates implies the same for solutions of equation (15). Now we may use the homogeneity of the vector field to extend the solutions to an arbitrarily small attractive neighborhood of the origin. The details are now presented.

Proof. We first recall the usual averaging results. The reader is referred to Hale [6] (Lemma V3.1, Lemma V3.2 and Lemma 5 of the appendix) For any compact set $\Omega$ in $\mathbf{R}^{n}$ there exists an $\epsilon_{0}$ and a function $u(t, x, \epsilon)$ such that the averaging transformation,

$$
x=y+\epsilon u(t, y, \epsilon) \quad(t, y, \epsilon) \in \mathbf{R} \times \Omega \times\left[0, \epsilon_{0}\right),
$$

applied to (15) yields the equation

$$
\dot{y}=\epsilon f_{0}(y)+\epsilon F(t, y, \epsilon),
$$

where $f_{0}$ is the averaged vector field as defined above. $F(t, y, \epsilon)$ is continuous for $(t, y, \epsilon) \in \mathbf{R} \times \Omega \times\left[0, \epsilon_{0}\right)$ and $F(t, y, 0)=0$. The function $u$ possesses the following properties on $R \times \Omega \times\left[0, \epsilon_{0}\right)$ :

1. $u(t, x, \epsilon)$ is periodic with period $2 \pi$ (same period as the vector field),

2. has continuous derivatives with respect to $t$ and derivatives of an arbitrary specified order with respect to $x$.

3. $\epsilon u$ and $\epsilon \frac{\partial u}{\partial y}$ approach 0 as $\epsilon \rightarrow 0$ uniformly in $t \in \mathbf{R}^{n}$ and $y \in \Omega$.

The solution $y=0$ of $(17)$ is asymptotically stable. Since this vector field is homogeneous with respect to $\delta_{\lambda}$ we may apply the recent results of Rosier [16] to obtain a Lyapunov function $V: R^{n} \rightarrow R$ with the following properties,

1. $V$ is as smooth as we require,

2. $V(0)=0, V(y)>0$ for all $y \neq 0$, and $V$ is radially unbounded,

3. $V$ is homogeneous with respect to the same dilation $\delta_{\lambda}$,

4. $\nabla V \cdot f_{0}(y)<0$ for all $x \neq 0$.

Consider the compact sets defined by

$$
D_{\alpha}=\left\{y \in \mathbf{R}^{n} \mid V(y) \leq \alpha\right\} . \quad \alpha>0
$$

The boundaries of these sets are denoted $\partial D_{\alpha}$. Given $D_{\alpha}$ we define constants

$$
\bar{\sigma}_{\alpha}=\max _{y \in \partial D_{\alpha}} \rho(y) \quad \underline{\sigma}_{\alpha}=\min _{y \in \partial D_{\alpha}} \rho(y) .
$$

Choose $c_{1}>0$ such that $D_{c_{1}} \subset \Omega$. Now find $c_{2}$, and corresponding $D_{c_{2}}$, such that $\bar{\sigma}_{D_{c_{2}}}<\underline{\sigma}_{D_{c_{1}}} / 2$. This may always be done because $V$ is positive definite and continuous. Evaluating $V$ along solutions of the transformed vector field (23) yields

$$
\frac{d V}{d t}=\epsilon \nabla V \cdot f_{0}(x)+\epsilon \nabla V \cdot F(t, y, \epsilon) \text {. }
$$

On the compact set $D_{c_{1}} \backslash D_{c_{2}}$ we calculate

$$
\beta=-\min _{y \in D_{c_{1}} \backslash D_{e}} \nabla V \cdot f_{0}(y) .
$$

Clearly $\beta>0$. We also define $M(\epsilon)$ as

$$
M(\epsilon)=\max _{y \in D_{c_{1}}, t \in S^{1}}|\nabla V \cdot F(t, y, \epsilon)| .
$$

$M(\epsilon)$ is continuous because $F$ is a continuous function of $\epsilon$ and $M(0)=0$ since $F(\cdot, \cdot, 0)=0$. The averaging transformation will not, in general, respect the dilation scaling. Hence the vector field $F(t, x, \epsilon)$ will not be homogeneous. For example we may be forced to bound $F$ with homogeneous functions of lower order than $f_{0}$ and hence asymptotic stability cannot be concluded with this Lyapunov analysis. On the annulus $D_{c_{1}} \backslash D_{c_{2}}$ the time derivative of $V$ is bounded by

$$
\frac{d V}{d t} \leq \epsilon(-\beta+M(\epsilon)) \text {. }
$$

Now choose $\tilde{\epsilon} \in\left(0, \epsilon_{0}\right)$ such that $M(\tilde{\epsilon}) \leq \frac{\beta}{2}$. The choice of $\tilde{\epsilon}$ renders $D_{c_{1}}$ and $D_{c_{2}}$ invariant. Trajectories through points in $D_{c_{1}} \backslash D_{c_{2}}$ will reach $D_{c_{2}}$ in a finite time no greater than

$$
\bar{T}=\frac{2\left(c_{1}-c_{2}\right)}{\tilde{\epsilon} \beta},
$$


because $\dot{V}<-\epsilon \beta / 2$ on $D_{\epsilon_{1}} \backslash D_{c_{2}}$. Choosing any $\epsilon \in(0, \tilde{\varepsilon})$ does not change the invariance or attractive nature of the sets. The only modifcation in this case is $\bar{T}$. The functional relationship of $\bar{T}$ is exactly the one given above with $\tilde{\epsilon}$ replaced by the new $\epsilon$. In the $y$-coordinates we can't say anything more about the stability of the zero solution. However, we may map the $D_{i}$ 's back to the extended phase space of (15) with the diffeomorphism (22). This will result in a warped version of $S^{1} \times D_{i}$ 's. We would like to bound these warped sets with homogeneous balls and apply the scaling lemma to conclude asymptotic stability. This is worked out in detail below.

Recall the map $x=y+\epsilon u(t, y, \epsilon)$ is a $C^{r}$ diffeomorphism for $(t, y, \epsilon) \in S^{1} \times \Omega \times\left[0, \epsilon_{0}\right)$. As $\epsilon \rightarrow 0$ this map approaches the identity. Since $u$ is $2 \pi$ periodic in $t$ it is useful to define the following diffeomorphism between $S^{1} \times \Omega$ and the extended phase space of the vector field in (15), $S^{1} \times R^{n}$,

$$
\varphi_{\epsilon}(t, y)=(t, y+\epsilon u(t, y, \epsilon)) .
$$

Define the compact sets in $S^{1} \times R^{n}$

$$
E_{c_{1}}=\varphi_{c}\left(t, D_{c_{1}}\right) \quad E_{c_{2}}=\varphi_{c}\left(t, D_{c_{2}}\right) .
$$

For fixed $t, E_{c_{1}} \rightarrow\left(t, D_{c_{\mathrm{i}}}\right)$ as $\epsilon \rightarrow 0$. The boundaries of $E_{c_{\mathrm{i}}}$ are denoted $\partial E_{c_{i}}$. As for the sets $D_{a}$, we define the quantities,

$$
\bar{\sigma}_{E_{c_{i}}}=\max _{(t, x) \in \partial E_{\varepsilon_{i}}} \rho(x) \quad \underline{\sigma}_{E_{c_{i}}}=\min _{(t, x) \in \partial E_{c_{i}}} \rho(x) .
$$

Note that

$$
\widetilde{\sigma}_{E_{c_{i}}} \rightarrow \bar{\sigma}_{D_{c_{i}}} \quad \underline{\sigma}_{E_{c_{i}}} \rightarrow \underline{\sigma}_{D_{c_{i}}},
$$

as $\epsilon \rightarrow 0$ since $\partial E_{c_{i}} \rightarrow\left(t, \partial D_{c_{i}}\right)$ for each $t \in S^{1}$. It is possible for $\bar{\sigma}_{E_{c_{2}}}>$ $\underline{\sigma}_{E_{c_{1}}}$ for the choice of $\tilde{\epsilon}$ made above (at different times of course). The relations in (24) imply $\epsilon$ may be further decreased to ensure $\bar{\sigma}_{E_{\varepsilon_{2}}}<\underline{\sigma}_{E_{c_{1}}}$ since $\bar{\sigma}_{E_{c_{2}}} \rightarrow \bar{\sigma}_{D_{c_{2}}}$ as $\epsilon \rightarrow 0$ (recall $\bar{\sigma}_{D_{c_{2}}}<\underline{\sigma}_{D_{e_{1}}} / 2$ from the choice of $c_{1}$ and $c_{2}$ ). Hence $\partial E_{c_{3}} \cap \partial E_{c_{1}}=\emptyset$. Now we may define homogeneous balls that are proper subsets of one another. Define the homogeneous balls in $S^{\mathbf{1}} \times \mathbf{R}^{\mathbf{n}}$,

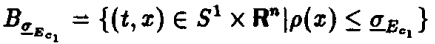

$$
\begin{aligned}
& B_{\bar{\sigma}_{\bar{s}_{c_{1}}}}=\left\{(t, x) \in S^{1} \times \mathbf{R}^{n} \mid \rho(x) \leq \bar{\sigma}_{E_{c_{1}}}\right\} \\
& B_{\bar{\sigma}_{\bar{s}_{c_{z}}}}=\left\{(t, x) \in S^{1} \times \mathbf{R}^{n} \mid \rho(x) \leq \bar{\sigma}_{E_{\sigma_{2}}}\right\} .
\end{aligned}
$$

The previous choice of $\epsilon$ leads to the following inclusions,

$$
E_{c_{2}} \subset B_{\bar{\sigma}_{E_{\sigma_{2}}}} \subset B_{\underline{\sigma}_{\sigma_{c_{1}}}} \subset E_{c_{1}} \subset B_{\bar{\sigma}_{\bar{\sigma}_{c_{1}}}} \text {. }
$$

Now we will say a few words about solutions with initial conditions in these sets. $E_{c_{1}}$ is invariant under (15) because $D_{c_{1}}$ is invariant under (23) and the diffeomorphism (22) takes $D_{c_{1}}$ into $E_{c_{1}}$. Furthermore, solutions of (15) with initial conditions in $E_{c_{1}}$ will reach the set $E_{c_{2}}$ in no less than time $\bar{T}$ and remain thereafter because these corresponding facts hold for $D_{c_{1}}$ and $D_{c_{2}}$ and the (23) maps $D_{c_{2}}$ to $E_{c_{2}}$. Hence, solutions through points in $B_{\underline{\sigma}_{E_{\varepsilon_{1}}}}$ are constrained to remain in $B_{\bar{\sigma}_{E_{\sigma_{1}}}}$ and furthermore must enter $B_{\bar{\sigma}_{\bar{E}_{\sigma_{2}}}}$ in finite time, $\bar{T}$, and remain there. Now apply Lemma 4.1 with $B_{1}=B_{\bar{\sigma}_{E_{c_{1}}}}, B_{2}=B_{{\underline{g_{\delta_{1}}}}_{1}}$ and $B_{3}=B_{\bar{\sigma}_{\varepsilon_{c_{2}}}}$ to conclude asymptotic stability of the zero solution.

Remark The same arguments may be used to show that trajectories moving from $B_{3}$ to the outer boundary of $B_{2}$ imply the origin is unstable.

\section{Conclusions}

This paper introduces some new tools for the analysis of nonholonomic systems as well as introducing useful ideas in the search for exponentially stabilizing controllers. A general stability analysis procedure was proposed and applied to power form equations with homogeneous feedbacks. The stability analysis involves two steps. The first step is a reduction to an invariant set. This was motivated by a "naive" perturbation scheme. The stability of the reduced equations on the invariant set was then performed using a modified averaging theorem. The usual invariant manifold theory cannot be applied directly because the systems are only $C^{0}$. Simulations indicate the possibility of proving some reduction results for these systems.
The idea of reduction seems to be a recurring theme in nonholonomic system analysis because the control inputs can directly stabilize only a few states in the phase space. The remaining directions are stabilized by "bracket" motions and occur at slower rates. For $C^{2}$ vector fields, this idea is embodied in the center manifold theory.

Homogeneous feedbacks were shown to be a nice choice for the $C^{0}$ stabilizing feedback. A key concept of the analysis is the equivalence of uniform asymptotic stability with exponential stability in the appropriate homogeneous norm.

\section{References}

[1] R. W. Brockett. Asymptotic stability and feedback stabilization. In R. W. Brockett, R. S. Millman, and H. J. Sussman, editors, Differential Geometric Control Theory, pages 181-191. Birkhauser, 1983.

[2] J-M. Coron. Global asymptotic stabilization for controllable systems without drift. Mathematics of Control, Signals, and Systems, 5:295-312, 1991

[3] J-M. Coron and J-B. Pomet. A remark on the design of timevarying stabilizing feedback laws for controllable systems without drift. In IFAC Symposium on Nonlinear Control Systems Design (NOLCOS), pages 413-417, Bordeaux, France, June 1992.

[4] C. Canudas de Wit and O.J. Sørdalen. Exponential stabilization of mobile robots with nonholonomic constraints. In IEEE Control and Decision Conference, pages 692-697, 1991.

[5] L. Gurvits and Z.X. Li. Smooth time-periodic feedback solutions for nonholonomic motion planning. Technical report, Courant In stitute of Mathematical Sciences, New York, 1992. (preprint).

[6] J. Hale. Ondinary Differential Equations. Wiley-Interscience, 1969.

[7] H. Hermes. Asymptotically stabilizing feedback controls. Journal of Differential Equations, 92(1):76-89, 1991.

[8] M. Kawski. Homogeneous stabilizing feedback laws. ControlTheory and Advanced Technology, 6(4):497-516, 1990.

[9] G. Lafferriere and H. J. Sussmann. Motion planning for controllable systems without drift. In IEEE International Conference on Robotics and Automation, pages 1148-1153, 1991.

[10] R. T. M'Closkey and R. M. Murray. Convergence rates for nonholonmic systems in power form. In American Control Conference, pages 2967-2972, 1992.

[11] R. M. Murray. Nilpotent bases for a class of non-integrable distributions with applications to trajectory generation for nonholonomic systems. Technical Report CIT/CDS 92-002, California Institute of Technology, October 1992.

[12] R. M. Murray and S. S. Sastry. Nonholonomic motion planning: Steering using sinusoids. IEEE Transactions on Automatic Control, 38(5):700-716, 1993

[13] R. M. Murray, G. Walsh, and S. S. Sastry. Stabilization and tracking for nonholonomic systems using time-varying state feedback. In IFAC Symposium on Nonlinear Control Systems Design (NOLCOS), Bordeaux, France, June 1992.

[14] J-B. Pomet. Explicit design of time-varying stabilizing control laws for a class of controllable systems without drift. Systems and Control Letters, 18(2):147-158, 1992.

[15] O. J. Sørdalen. Feedback Control of Nonholonomic Mobile Robots. $\mathrm{PhD}$ thesis, The Norwegian Institute of Technology, Trondheim, Norway, 1993.

[16] L. Rosier. Homogeneous lyapunov function for homogeneous continuous vector field. Systems and Control Letters, 19(6):467-473, 1992.

[17] C. Samson and K. Kit-Abderrahim. Feedback stabilization of a nonholonomic wheeled mobile robot. In International Conference on Intelligent Robots and Systems (IROS), 1991.

[18] A. Teel, R. M. Murray, and G. Walsh. Nonholonomic control systems: From steering to stabilization with sinusoids. In IEEE Control and Decision Conference, pages 1603-1609, 1992. 